\title{
Erreurs médicamenteuses en lien avec l'insuline
}

\author{
Olga Frank ${ }^{a}$, Larissa Gehrig ${ }^{b}$, David Schwappach
}

${ }^{a}$ Dr sc. hum., responsable Projets, produits et services Fondation pour la Sécurité des patients Suisse; ${ }^{b}$ cand. MscN; ${ }^{c}$ Prof. Dr, MPH, directeur scientifique

\section{Résumé}

Les erreurs médicamenteuses surviennent à toutes les étapes du circuit du médicament. Sans conséquence dans certains cas, elles peuvent aussi occasionner de graves préjudices aux patients. L'insuline est un médicament à haut risque dont l'utilisation requiert des précautions particulières. La fondation Sécurité des patients Suisse a procédé à une recherche systématique dans la banque de données CIRRNET, analysé les rapports décrivant des erreurs en lien avec l'insuline et identifié sur cette base des problématiques spécifiques. Si les erreurs médicamenteuses impliquant l'insuline concernent toutes les étapes du circuit du médicament, les signalements les plus fréquents portent sur son administration. Les problèmes mis en évidence à ce niveau sont essentiellement des erreurs de dosage, des omissions et des confusions de médicaments. La gestion des patients diabétiques à l'hôpital représente un défi particulier, du fait que l'insuline doit être administrée sans tarder en fonction de la glycémie et d'autres facteurs et que son dosage nécessite des adaptations régulières.

\section{Contexte}

La prise en charge de patients diabétiques faisant partie du quotidien clinique de tout hôpital, elle tend à prendre un caractère routinier qui suscite un sentiment de sécurité trompeur, surtout dans les cas où le diabète n'est pas la raison première du séjour. Les patients multimorbides, en particulier, courent le risque de voir le traitement médical de leurs diagnostics secondaires négligé par rapport à celui que nécessite le diagnostic principal ayant motivé l'hospitalisation. Les erreurs qui peuvent alors se produire - omettre de contrôler régulièrement la glycémie chez un patient diabétique avant une intervention planifiée ou oublier d'administrer l'insuline avant un repas, par exemple sont susceptibles de déséquilibrer la glycémie, ce qui peut avoir de graves conséquences pour le patient.

L'insuline fait partie des médicaments à haut risque [1]. Elle possède un index thérapeutique étroit et requiert des adaptations précises du dosage, une administration prudente et des contrôles réguliers. En outre, il existe dans le commerce toute une série de dispositifs d'injection (p. ex. seringues, stylos, pompes à insuline, etc.) ainsi que plusieurs types d'insulines de formes et concentrations différentes, ce qui rend l'emploi correct de ce médicament particulièrement complexe.

Face à l'importance des erreurs médicamenteuses impliquant l'insuline, la fondation Sécurité des patients Suisse a décidé de procéder à une analyse systématique des rapports enregistrés dans CIRRNET (Critical Incident Reporting \& Reacting NETwork) portant sur des erreurs en lien avec ce médicament. Nous présentons ci-après les notifications décrivant une problématique concernant l'insuline selon les étapes du circuit du médicament (Circuit du médicament: 1. prescription, 2. documentation, 3. délivrance/préparation, 4. administration, 5. Suivi) [2] et indiquons pour chacune d'elles les problèmes fréquents mis en évidence.

\section{Méthode}

CIRRNET est un réseau suisse de systèmes locaux de déclaration des erreurs exploité depuis 2006 par la Fondation Sécurité des patients Suisse. A l'aide de mots-clés définis, une recherche systématique a été menée en avril 2015 dans la banque de données CIRRNET afin d'identifier les déclarations d'erreurs en lien avec l'insuline. Au total, 267 rapports ont été trouvés et 195 ont été retenus comme pertinents au terme d'un premier examen. Les notifications non prises en compte ont été exclues pour les raisons suivantes: elles n'étaient pas directement liées à la problématique de l'insuline, elles étaient mentionnées à double ou les mots-clés choisis y apparaissaient uniquement sous forme d'exemples. Deux reviewers travaillant indépendamment l'un de l'autre ont classé les déclarations d'erreurs retenues selon les étapes du circuit du médicament et identifié les problématiques décrites. Les informations contenues dans les rapports étant très limitées, il s'est avéré difficile de savoir avec certitude à quelle catégorie attribuer les erreurs en fonction du circuit du médicament ainsi que de la problématique évoquée. Les reviewers ont donc décidé de commencer par tester la classification sur un échantillon choisi de façon aléatoire et de débattre ensemble des résultats pour s'accorder sur la 
façon de classer les rapports. Ils ont ensuite procédé, chacun de son côté, à la classification et à l'analyse de l'ensemble des déclarations d'erreurs retenues.

\section{Résultats}

L'analyse des rapports CIRRNET en lien avec l'insuline s'est révélée concordante dans $80 \%$ des cas $(n=212)$ pour la classification en fonction du circuit du médicament. Les divergences ont été clarifiées a posteriori d'entente entre les deux reviewers. Le nombre de notifications CIRRNET par étape du circuit du médicament ainsi que les problématiques mises en évidence sont présentés en détail dans le tableau 1 .

Pour la première étape, à savoir la prescription (13,3\%), sept problèmes ont été identifiés à partir de 26 déclara- tions d'erreurs. Les plus fréquents sont l'absence de prescription d'insuline (3,6\%), ainsi que les prescriptions imprécises $(3,1 \%)$ contenant par exemple des indications contradictoires relatives au débit de perfusion. En ce qui concerne les schémas pour les injections complémentaires, on relève en particulier le problème des prescriptions multiples (2,6\%), dont le contenu ne concorde pas et/ou qui sont établies plusieurs fois pour un seul et même patient. Il est également fait mention de prescriptions illisibles et/ou incomplètes, mais aussi de l'absence de communication par le médecin à l'équipe soignante d'une nouvelle prescription ou d'une prescription modifiée.

Les rapports enregistrés dans CIRRNET signalent également plusieurs types d'erreurs concernant l'étape de la documentation (9,7\%). Il s'agit notamment d'erreurs de

Tableau 1: Classification en fonction du circuit du médicament et des problématiques décrites.

\begin{tabular}{|c|c|c|c|c|c|}
\hline Etape & Problématique & $\begin{array}{l}\text { Nombre } \\
\text { de cas }\end{array}$ & Total & $\%$ de cas & Total \\
\hline \multirow[t]{7}{*}{ 1. Prescription } & absence de prescription & 7 & 26 & $3,59 \%$ & $13,3 \%$ \\
\hline & prescription imprécise & 6 & & $3,08 \%$ & \\
\hline & prescription illisible & 1 & & $0,51 \%$ & \\
\hline & prescription incomplète & 1 & & $0,51 \%$ & \\
\hline & prescription incorrecte & 5 & & $2,56 \%$ & \\
\hline & prescription multiple & 5 & & $2,56 \%$ & \\
\hline & non-communication d'une nouvelle prescription ou d'une modification & 1 & & $0,51 \%$ & \\
\hline \multirow[t]{7}{*}{ 2. Documentation } & absence de documentation & 5 & 19 & $2,56 \%$ & $9,7 \%$ \\
\hline & documentation imprécise & 2 & & $1,03 \%$ & \\
\hline & documentation illisible & 1 & & $0,51 \%$ & \\
\hline & documentation incomplète & 3 & & $1,54 \%$ & \\
\hline & documentation incorrecte & 2 & & $1,03 \%$ & \\
\hline & erreur de transcription & 5 & & $2,56 \%$ & \\
\hline & inexpérience dans I'utilisation du système de documentation électronique & 1 & & $0,51 \%$ & \\
\hline \multirow[t]{4}{*}{ 3. Délivrance/Préparation } & préparation incorrecte & 1 & 7 & $0,51 \%$ & $3,6 \%$ \\
\hline & médicament erroné & 3 & & $1,54 \%$ & \\
\hline & absence d'étiquetage & 1 & & $0,51 \%$ & \\
\hline & erreur de calcul & 2 & & $1,03 \%$ & \\
\hline \multirow[t]{10}{*}{ 4. Administration } & erreur de dosage & 36 & 132 & $18,46 \%$ & $67,7 \%$ \\
\hline & confusion de médicaments & 27 & & $13,85 \%$ & \\
\hline & omission & 36 & & $18,46 \%$ & \\
\hline & non-respect de prescriptions & 8 & & $4,10 \%$ & \\
\hline & double administration & 1 & & $0,51 \%$ & \\
\hline & erreur d'horaire d'administration & 4 & & $2,05 \%$ & \\
\hline & erreur d'identification du patient & 14 & & $7,18 \%$ & \\
\hline & erreur en cas d'automédication & 4 & & $2,05 \%$ & \\
\hline & incompatibilité entre médicaments & 1 & & $0,51 \%$ & \\
\hline & mode d'administration incorrect (SC/IV) & 1 & & $0,51 \%$ & \\
\hline \multirow[t]{4}{*}{ 5. Suivi } & $\begin{array}{l}\text { absence d'adaptation adéquate en cas de modification de l'état clinique } \\
\text { du patient }\end{array}$ & 3 & 11 & $1,54 \%$ & $5,6 \%$ \\
\hline & gestion déficiente ou retardée lors de l'admission de patients diabétiques & 5 & & $2,56 \%$ & \\
\hline & utilisation d'un équipement incorrect & 2 & & $1,03 \%$ & \\
\hline & $\begin{array}{l}\text { manque/absence de communication de résultats et de prescriptions } \\
\text { aux interfaces }\end{array}$ & 1 & & $0,51 \%$ & \\
\hline Total & & 195 & 195 & $100 \%$ & $100 \%$ \\
\hline
\end{tabular}


transcription (2,6\%), fréquentes avec les systèmes de documentation non informatisés. Comme le montrent les déclarations d'erreurs saisies dans CIRRNET, les systèmes électroniques ne permettent cependant pas d'éviter le problème de l'absence de documentation (2,6\%). La majorité des rapports CIRRNET analysés portent sur des erreurs relatives à l'étape de l'administration de l'insuline (67,7\%). Les plus fréquentes sont les erreurs de dosage $(18,5 \%)$ - surdosage ou sous-dosage - ainsi que les omissions (18,5\%). Les erreurs de dosage décrites couvrent des situations très diverses. Par exemple, pour l'insuline «Novomix 30», le chiffre figurant dans le nom du médicament a par erreur été compris comme la mention du dosage dans la documentation manuscrite et le médicament a été administré selon cette indication. Quant aux omissions, elles incluent notamment l'oubli d'administrer l'insuline dans le cadre d'une délégation de tâche ou en raison d'une surcharge de travail. Il est également fait état de confusions (13,9\%) entre des insulines rapides et des insulines lentes, ainsi qu'entre des insulines à consonance semblable, telles que " Levemir» et "Lantus». De plus, les erreurs d'identification ne portent pas que sur les médicaments, mais aussi sur les patients. Ainsi, 14 notifications $(7,2 \%)$ rapportent des situations où l'on se trompe de patient: c'est le voisin qui reçoit l'insuline ou celle-ci est administrée à un patient qui ne présente pas d'indication pour ce médicament (personne non diabétique). Les rapports mentionnent en outre des erreurs d'horaire d'administration $(2,1 \%)$ ainsi que divers problèmes concernant les patients qui s'injectent eux-mêmes leur insuline durant leur séjour à l'hôpital (2,1\%). L'analyse des déclarations d'erreurs enregistrées dans CIRRNET fournit par ailleurs des résultats intéressants au sujet de la gestion des admissions de patients diabétiques. Il est notamment signalé des cas d'omission de prescription ou de retard dans la prescription du traitement médicamenteux pour des patients hospitalisés avec un diabète sucré comme diagnostic secondaire (2,6\%). Il est également fait mention de réactions tardives à une modification aiguë de l'état clinique du patient en raison d'une hypoglycémie ou d'une hyperglycémie par exemple (1,5\%). L'adaptation précise et immédiate du dosage selon les variations du taux de glucose dans le sang et, partant, la surveillance rapprochée et régulière de la glycémie représentent un défi majeur dans le quotidien hospitalier, en particulier lorsque le personnel de l'établissement est soumis à une très forte charge de travail.

\section{Conclusion}

La présente analyse avait pour but de procéder à une revue systématique des rapports enregistrés dans
CIRRNET concernant des erreurs en lien avec l'insuline et d'identifier des problématiques spécifiques relatives à son utilisation. Ses résultats concordent avec ceux obtenus par d'autres institutions à l'échelle internationale. Une analyse du National Reporting and Learning System [3] en Grande-Bretagne, notamment, a montré que les problèmes principaux liés à l'emploi de l'insuline étaient les erreurs de dosage, l'omission d'administration ou les confusions entre médicaments. Tous ces éléments se rapportent à l'étape de l'administration de l'insuline dans le circuit du médicament.

Concernant l'interprétation des résultats, il est important de souligner que l'on ne connaît pas le groupe professionnel à l'origine des notifications CIRRNET analysées, ce qui peut introduire un certain biais. Ces données livrent néanmoins des informations de toute première importance pour la gestion des risques et de la qualité ainsi que pour une meilleure sécurité des patients au niveau de l'utilisation de l'insuline. En ce sens, les cas isolés relatant des incidents inhabituels permettent de repérer des situations certes rares mais susceptibles de causer de graves préjudices, et de prendre à cet égard des mesures correctives. Le problème mentionné dans le rapport concernant le "Novomix 30", dont le nom a porté à confusion, n'aurait peut-être jamais pu être identifié par un autre moyen. Les omissions, en revanche, sont connues des professionnels, ce que confirme la fréquence des déclarations d'erreurs se rapportant à cette problématique. Ce type d'erreur est particulièrement souvent mis en lien avec une charge de travail élevée. S'ajoute à cela le fait que, l'administration de l'insuline étant fonction du taux de glycémie du moment, la mesure de cette valeur doit coïncider au plus près avec le calcul de la dose d'insuline et son administration. En période de surcharge de travail, il n'est pas rare que cette médication soit oubliée.

Une attention particulière devrait être accordée à la gestion des admissions de patients diabétiques hospitalisés pour un autre diagnostic principal. En tant que diagnostic secondaire, le diabète sucré n'est pas assez pris en compte, alors qu'il ne peut souffrir de retard de prescription ni de traitement.

Des améliorations sont nécessaires au niveau de l'utilisation de l'insuline et de sa gestion chez les patients diabétiques. Les résultats de l'analyse des rapports CIRRNET fournissent à cet égard des indications sur les problèmes existants. Tous les établissements de santé devraient passer en revue les modalités d'emploi de l'insuline afin de combler les lacunes éventuelles et de contribuer à accroître la sécurité de la médication et la sécurité des patients. 